\title{
Post-Marketing Surveillance of Silodosin in Patients with Benign Prostatic Hyperplasia and Poor Response to Existing Alpha-1 Blockers: The SPLASH Study
}

\author{
Hiroshi Takahashi ${ }^{1} \cdot$ Shinichi Kubono ${ }^{1} \cdot$ Takehiko Taneyama $^{2} \cdot$ Kiyotoshi Kuramoto $^{2} \cdot$ Hideki Mizutani $^{3}$. \\ Noriko Tanaka ${ }^{3} \cdot$ Masaki Yoshida $^{4}$
}

Published online: 3 January 2019

(c) The Author(s) 2018

\begin{abstract}
Objectives Our objective was to investigate the effectiveness and safety of silodosin in patients with benign prostatic hyperplasia (BPH) who switched to silodosin from another $\alpha_{1}$ blocker because of inadequate response.

Methods This was a prospective observational study conducted at 715 medical facilities in Japan in patients with BPH who received an $\alpha_{1}$ blocker other than silodosin for at least 3 months but had experienced unsatisfactory treatment outcomes. Patients completed questionnaires, including the International Prostate Symptom Score (IPSS), quality of life (QOL) score and Overactive Bladder Symptom Score (OABSS) at baseline (time of switching) and after 3 months of treatment with silodosin. Results Overall, 3355 patients were assessed for safety and 3144 patients for effectiveness. Mean \pm standard deviation age was $73.1 \pm 8.2$ years, and most patients had been receiving tamsulosin (53.6\%) or naftopidil (45.5\%) before silodosin. Silodosin was well tolerated, with an overall incidence of adverse drug reactions of $8.1 \%$ and no unexpected safety signals. Significant improvements were observed after switching to silodosin in all effectiveness outcome measures, including total IPSS, all IPSS subscale scores, QOL score, total OABSS, all OABSS subscale scores and residual urine volume. Significant improvements in total IPSS were seen in patients who had been receiving tamsulosin or naftopidil before switching and in almost all other patient subgroups, with the exception of patients with mild symptoms (total IPSS $\leq 7$ ) at baseline.

Conclusions This post-marketing analysis indicates that switching to silodosin from tamsulosin or naftopidil significantly improved symptoms associated with BPH, and silodosin was well tolerated in Japanese patients.
\end{abstract}

\section{Key Points}

A prospective observational study was conducted in Japan to investigate the effectiveness and safety of silodosin in patients with benign prostatic hyperplasia.

Silodosin showed favorable safety profiles and significant improvement in Japanese patients, including International Prostate Symptom Scores, quality of life scores and Overactive Bladder Symptom Scores.

Hiroshi Takahashi

hiroshi_takahashi@pharm.kissei.co.jp

1 Medical Research Department, Kissei Pharmaceutical Co., Ltd., 1-8-9 Nihonbashi Muromachi, Chouo-ku, Tokyo 103-0022, Japan

2 Pharmacovigilance and Post-Marketing Surveillance Department, Kissei Pharmaceutical Co., Ltd., Tokyo, Japan

\section{Introduction}

Silodosin (Urief ${ }^{\circledR}$, Kissei Pharmaceutical Co. Ltd. and Daiichi Sankyo Co. Ltd.) is a selective $\alpha_{1 \mathrm{~A}}$ blocker developed in Japan and has been on the market since May 2006. Two post-marketing surveys (a drug use results survey [1] and a specified drug use results survey [2]) were conducted after launch, followed by the submission of a reexamination application in April 2014, and the results were reported in June 2015 .

Pharmacovigilance Department, Daiichi Sankyo Co., Ltd., Tokyo, Japan

4 Department of Urology, National Center for Geriatrics and Gerontology, Obu, Japan 
The number of patients with benign prostatic hyperplasia (BPH) is expected to increase in Japan, which is becoming a super-aging society. As a result, physicians will be seeing more patients with BPH with age-related progression of symptoms and prolonged periods of treatment and will need to select the optimal drug based on the background of each individual patient. Currently in Japan, $\alpha_{1}$ blockers, including silodosin, are recommended in the Clinical Guidelines for BPH in Japan as a first-line drug therapy for lower urinary tract symptoms (LUTS) associated with BPH [3], but selection of an $\alpha_{1}$ blocker depends on the preference of each physician. Furthermore, clinical evidence about switching between $\alpha_{1}$ blockers is currently limited.

A pooled analysis of the earlier post-marketing surveys $[1,2]$ with silodosin included an assessment of patients with a treatment history of $\alpha_{1}$ blockers other than silodosin and a low degree of satisfaction (quality of life (QOL) score $\geq 3$ points) who switched to silodosin [4]. The results suggested that the voiding symptom score of the International Prostate Symptom Score (IPSS) was an influential factor on the effectiveness of silodosin in LUTS associated with BPH.

SPLASH (Study on patients' QOL by changing medication to silodosin in men with BP$\underline{H}$ ) was a post-marketing surveillance study designed to identify an appropriate patient profile for switching to silodosin. It was a prospective observational study to complement the pooled analysis of the two earlier post-marketing surveys and included the Overactive Bladder Symptom Score (OABSS) in addition to the IPSS and QOL score evaluations included in the previous analyses.

The survey was conducted in accordance with good postmarketing study practice in Japan.

\section{Methods}

\subsection{Study Population}

The study population was selected from among patients with BPH who had not experienced satisfactory outcomes with $\alpha_{1}$ blockers other than silodosin in routine clinical practice and according to the following inclusion criteria: patients who (1) had not received silodosin previously, (2) gave consent to change their medication from their current $\alpha_{1}$ blocker to silodosin, (3) had been using another $\alpha_{1}$ blocker other than silodosin for at least 3 months and (4) were evaluated using the face scale questionnaire before the start of silodosin administration. Exclusion criteria were patients who (1) had a QOL score of $\leq 2$ before the start of silodosin administration and/or (2) had started treatment with a $5 \alpha$-reductase inhibitor within 6 months before the start of silodosin administration.
Patients were enrolled using a prospective central registration method through an online system. The enrollment period was for 1 year from 1 November 2014 to 31 October 2015 , and the observation period was set for 3 months. The targeted number of patients for the survey was 3000 .

\subsection{Survey Items}

Basic information collected in the study included patient background, administration status of silodosin, and drug therapy history for LUTS other than silodosin (including prior or concomitant therapies).

Patients were also asked to complete a symptoms checklist before the start of administration and at 3 months after the start of administration (or at the final evaluation) so we could understand the patients' LUTS. The IPSS, the QOL score, and the OABSS were obtained through the symptoms checklist. In addition, residual urine volume was measured before the start of administration and at 3 months after the start of administration (or at the final evaluation) as an objective outcome measure.

Safety data collected in the study included the occurrence of adverse events (AEs) and the likely causal relationship with silodosin in the case of onset of an AE.

\subsection{Statistical Analysis}

Baseline demographic and clinical information was summarized using descriptive summary statistics, including frequency for categorical variables and mean \pm standard deviation (SD) for continuous variables.

For the safety assessment, the incidence of adverse drug reactions (ADRs), defined as AEs for which a causal relationship with silodosin could not be ruled out, was calculated for the whole cohort and in specific patient subgroups based on background factors. ADRs were classified according to the preferred terms in the Medical Dictionary for Regulatory Activities-Japanese, version 19.0.

Effectiveness was assessed as the change from baseline in the overall cohort and in subgroups based on the $\alpha_{1}$ blocker (tamsulosin or naftopidil) administered prior to silodosin.

The impact of background factors on the incidence of ADRs was assessed using the chi-squared test and Fisher's exact test, depending on the data properties. To compare the effectiveness measurements before and after administration, a one-sample $t$-test was used. The two-tailed significance level was set at $5 \%$. 


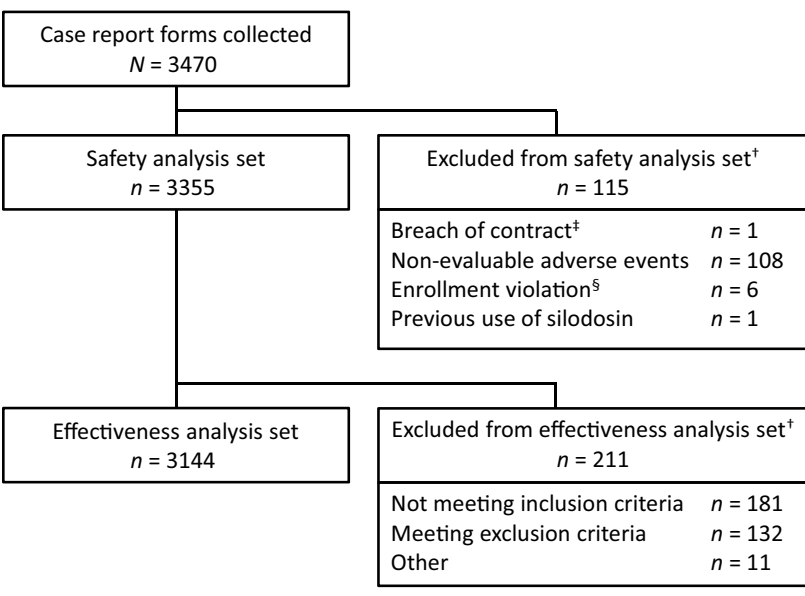

Fig. 1 Patient disposition. ${ }^{\dagger}$ Patients may be included more than once if they were excluded for more than one reason. ${ }^{\ddagger}$ Patients who received silodosin outside the contract period. ${ }^{\S}$ Patients who were not registered within the registration period

\section{Results}

\subsection{Case Description}

Figure 1 shows the patient disposition in the study. A total of 3470 case report forms were obtained from 715 medical facilities across Japan. Of those, 115 cases were excluded for reasons such as breach of contract, non-evaluable AEs, enrollment violations, and previous use of silodosin, leaving 3355 cases for the safety analyses. An additional 211 patients were excluded (not meeting inclusion criteria, meeting exclusion criteria, and other), leaving 3144 patients for the effectiveness analyses.

\subsection{Patient Background}

Baseline demographics and clinical characteristics of the patients in the safety cohort $(n=3355)$ are shown in Table 1 . Most patients (46.1\%) were aged $\geq 70$ and $<80$ years, and the mean age was $73.1 \pm 8.2$ years. The disease duration with the highest proportion was $\geq 3$ years $(48.9 \%)$. The most administered $\alpha_{1}$ blockers before silodosin were tamsulosin (53.6\%) and naftopidil (45.5\%), and other concomitant drugs used to treat LUTS included $5 \alpha$-reductase inhibitor (9.9\%), anticholinergics (8.5\%), $\beta_{3}$ agonist (7.0\%) and phosphodiesterase 5 (PDE5) inhibitor (2.1\%). Based on the total IPSS, most patients had moderate $(56.1 \%)$ or severe (31.9\%) symptoms, with only $8.4 \%$ having mild symptoms, and the mean total IPSS was $16.6 \pm 6.7$. The QOL score showed moderate or severe impairment in $63.3 \%$ and $33.6 \%$ of patients, respectively, and the mean QOL score was $4.2 \pm 0.9$. Total OABSS scores were in the mild category for $51.5 \%$ of
Table 1 Baseline demographics and clinical characteristics (safety analysis set)

\begin{tabular}{|c|c|}
\hline Items and categories & Patients $^{\mathrm{a}}(n=3355)$ \\
\hline \multicolumn{2}{|l|}{ Age (years) } \\
\hline$<50$ & $14(0.4)$ \\
\hline$\geq 50$ and $<60$ & $166(4.9)$ \\
\hline$\geq 60$ and $<70$ & $871(26.0)$ \\
\hline$\geq 70$ and $<80$ & $1545(46.1)$ \\
\hline$\geq 80$ & $759(22.6)$ \\
\hline Non-elderly patients: $<65$ & $485(14.5)$ \\
\hline Elderly patients: $\geq 65$ & $2870(85.5)$ \\
\hline Mean \pm SD & $73.1 \pm 8.2$ \\
\hline \multicolumn{2}{|l|}{ BMI $\left(\mathrm{kg} / \mathrm{m}^{2}\right)$} \\
\hline$<18.5$ & $85(2.5)$ \\
\hline$\geq 18.5$ and $<25.0$ & $1339(39.9)$ \\
\hline$\geq 25.0$ and $<30.0$ & $546(16.3)$ \\
\hline$\geq 30.0$ & $46(1.4)$ \\
\hline Unknown/unlisted & $1339(39.9)$ \\
\hline Mean \pm SD & $23.45 \pm 3.05$ \\
\hline \multicolumn{2}{|l|}{ Prostate volume $(\mathrm{mL})$} \\
\hline$<40$ & $1881(56.1)$ \\
\hline$\geq 40$ & $1083(32.3)$ \\
\hline Unknown/unlisted & $391(11.7)$ \\
\hline Mean \pm SD & $38.42 \pm 20.19$ \\
\hline \multicolumn{2}{|l|}{$\mathrm{BPH}$ duration } \\
\hline$<3$ months & $15(0.4)$ \\
\hline$\geq 3$ months and $<1$ year & $612(18.2)$ \\
\hline$\geq 1$ year and $<3$ years & $945(28.2)$ \\
\hline$\geq 3$ years & $1640(48.9)$ \\
\hline Unknown/unlisted & $143(4.3)$ \\
\hline \multicolumn{2}{|l|}{ PSA (ng/mL) } \\
\hline$\leq 4.0$ & $2305(68.7)$ \\
\hline$>4.0$ and $\leq 10.0$ & $659(19.6)$ \\
\hline$>10.0$ & $93(2.8)$ \\
\hline Unknown/unlisted & $298(8.9)$ \\
\hline Mean \pm SD & $3.377 \pm 16.940$ \\
\hline \multicolumn{2}{|l|}{ Complications } \\
\hline No & $1235(36.8)$ \\
\hline Yes $^{\mathrm{b}}$ & $2117(63.1)$ \\
\hline Hypertension & $1263(37.6)$ \\
\hline Dyslipidemia & $476(14.2)$ \\
\hline Diabetes mellitus & $415(12.4)$ \\
\hline Gout & $38(1.1)$ \\
\hline Hyperuricemia & $181(5.4)$ \\
\hline Heart disorder & $314(9.4)$ \\
\hline Unknown/unlisted & $3(0.1)$ \\
\hline \multicolumn{2}{|c|}{$\alpha_{1}$ Blockers used before silodosin } \\
\hline Tamsulosin & $1799(53.6)$ \\
\hline Naftopidil & $1525(45.5)$ \\
\hline Other & $19(0.6)$ \\
\hline
\end{tabular}


Table 1 (continued)

\begin{tabular}{ll}
\hline Items and categories & \multicolumn{1}{c}{ Patients $^{\mathrm{a}}(n=3355)$} \\
\hline Concomitant drugs for LUTS other than $\alpha_{1}$ blockers \\
No & $2195(65.4)$ \\
Yes ${ }^{\mathrm{b}}$ & $1160(34.6)$ \\
Anticholinergics & $285(8.5)$ \\
$5 \alpha$-Reductase inhibitor & $331(9.9)$ \\
PDE5 inhibitor & $69(2.1)$ \\
$\beta_{3}$ Agonist & $234(7.0)$ \\
Total IPSS & \\
Mild: $0-7$ & $281(8.4)$ \\
Moderate: $8-19$ & $1883(56.1)$ \\
Severe: $20-35$ & $1070(31.9)$ \\
Mean \pm SD & $16.6 \pm 6.7$ \\
QOL score & \\
Mild: $0-1$ & $1(0.0)$ \\
Moderate: $2-4$ & $2125(63.3)$ \\
Severe: $5-6$ & $1128(33.6)$ \\
Mean \pm SD & $4.2 \pm 0.9$ \\
Total OABSS & \\
Mild: $\leq 5$ & $1728(51.5)$ \\
Moderate: $6-11$ & $1349(40.2)$ \\
Severe: $\geq 12$ & $134(4.0)$ \\
Mean \pm SD & $5.6 \pm 3.0$ \\
Residual urine volume $(\mathrm{mL})$ & \\
$<50$ & $1361(40.6)$ \\
$\geq 50$ and $<100$ & $497(14.8)$ \\
$\geq 100$ & $332(9.9)$ \\
Unknown/unlisted & $1165(34.7)$ \\
Mean \pm SD & $52.4 \pm 64.7$ \\
\hline & \\
\hline &
\end{tabular}

$B M I$ body mass index, $B P H$ benign prostatic hyperplasia, IPSS International Prostate Symptom Score, LUTS lower urinary tract symptoms, OABSS Overactive Bladder Symptom Score, PDE phosphodiesterase, $P S A$ prostate-specific antigen, $Q O L$ quality of life, $S D$ standard deviation

${ }^{a}$ Data are presented as $n(\%)$ or mean \pm SD

${ }^{b}$ Patients may be included more than once if they had more than one condition or were taking more than one concomitant medication

patients, moderate for $40.2 \%$, and severe for $4.0 \%$, with a mean total OABSS of $5.6 \pm 3.0$.

\subsection{Safety}

\subsubsection{Incidence of Adverse Drug Reactions (ADRs)}

The overall incidence of ADRs and those that occurred in five or more patients are described in Table 2. Of the 3355 patients included in the safety analysis, 271 patients developed an ADR (8.1\%). ADRs occurring in $\geq 0.5 \%$ of patients included ejaculation disorder (1.5\%), retrograde ejaculation $(1.4 \%)$, diarrhoea (1.0\%), dizziness $(0.9 \%)$, and nasal congestion $(0.5 \%)$.
Table 2 Incidence of adverse drug reactions

\begin{tabular}{ll}
\hline ADRs & $\begin{array}{l}\text { Safety } \\
\text { analysis set } \\
(n=3355)\end{array}$ \\
\hline Number of patients developing $\geq 1$ ADR & 271 \\
Number of ADRs reported & 306 \\
Incidence of ADRs $(\%)$ & 8.1 \\
Specific ADRs occurring in $\geq 5$ patients, $n(\%)$ & \\
Ejaculation disorder & $52(1.5)$ \\
Retrograde ejaculation & $47(1.4)$ \\
Diarrhoea & $35(1.0)$ \\
Dizziness & $31(0.9)$ \\
Nasal congestion & $18(0.5)$ \\
Faeces soft & $13(0.4)$ \\
Dizziness postural & $11(0.3)$ \\
Thirst & $7(0.2)$ \\
Abdominal discomfort & $6(0.2)$ \\
Pollakiuria & $6(0.2)$ \\
Orthostatic hypotension & $5(0.1)$ \\
Urinary incontinence & $5(0.1)$ \\
\hline
\end{tabular}

ADRs adverse drug reactions

\subsubsection{Serious ADRs}

Two patients developed serious ADRs: haematemesis $(n=1)$ and urinary retention $(n=1)$. The patient with haematemesis died, and the investigator physician could not determine whether this was causally related to silodosin as the details of the death were unspecified. The urinary retention resolved after transurethral resection of the prostate; the investigator physician determined the causal relationship with silodosin as "unlikely".

\subsubsection{Incidence of ADRs Based on Patient Background Factors}

The incidence of ADRs based on patient background factors is described in Table 3. The age-specific incidence of ADRs was $17.1 \%$ in non-elderly (aged $<65$ years) and $6.6 \%$ in elderly (aged $\geq 65$ years) patients, with a significant difference between groups $(P<0.0001)$. The incidence of ADRs did not differ significantly between patients who used concomitant drugs for LUTS other than $\alpha_{1}$ blockers (7.6\%) and those who did not (8.3\%; $P=0.4644)$. Among patients who were taking concomitant therapy for LUTS, ADRs occurred in $7.7 \%$ of those taking anticholinergics, $6.9 \%$ of those taking a $5 \alpha$-reductase inhibitor, $10.1 \%$ of those taking a PDE5 inhibitor, and $5.6 \%$ of those taking a $\beta_{3}$ agonist. 
Table 3 Incidence of adverse drug reactions in patient subgroups according to background factors

\begin{tabular}{lccc}
\hline Items and categories & $n$ & ADRs, $\mathrm{n}(\%)$ & $p$ value \\
\hline All patients & 3355 & $271(8.1)$ & \\
\hline Age (years) & 14 & $3(21.4)$ & $<0.0001$ (Chi-squared) \\
$<50$ & 166 & $36(21.7)$ & \\
$\geq 50$ and $<60$ & 871 & $104(11.9)$ & $<0.0001$ (Fisher) \\
$\geq 60$ and $<70$ & 1545 & $102(6.6)$ & \\
$\geq 70$ and $<80$ & 759 & $26(3.4)$ & 0.4644 (Fisher) \\
$\geq 80$ & 485 & $83(17.1)$ & \\
\hline Non-elderly patients: $<65$ & 2870 & $188(6.6)$ & \\
Elderly patients: $\geq 65$ & 2195 & $183(8.3)$ & \\
Concomitant drugs for LUTS other than $\alpha_{1}$ blockers & $88(7.6)$ & \\
No & 1160 & $22(7.7)$ & \\
Yes & 285 & $23(6.9)$ & \\
\hline Anticholinergics & 331 & $13(10.1)$ & \\
$5 \alpha-$ Reductase inhibitor & 69 & 234 &
\end{tabular}

$A D R$ adverse drug reaction, LUTS lower urinary tract symptoms, $P D E$ phosphodiesterase

\subsection{Effectiveness}

\subsubsection{Improvement in International Prostate Symptom Score (IPSS)}

Overall, 1972-2510 patients in the effectiveness analysis set $(n=3144)$ had data for each symptom of the IPSS before and after the administration of silodosin, and the value for those symptoms was not 0 (Table 4 ). The mean total IPSS value before silodosin administration was $16.6 \pm 6.7$ but improved to $12.5 \pm 6.4$ after silodosin treatment $(P<0.0001)$. The voiding symptom score, the storage symptom score, and seven other symptoms in the IPSS questionnaire also showed significant improvements with silodosin treatment $(P<0.0001)$. Significant improvements in the mean total IPSS and all IPSS subscale scores were seen during silodosin therapy, regardless of whether patients had been receiving tamsulosin or naftopidil $(P<0.0001)$ prior to switching to silodosin.

\subsubsection{Improvement in Quality of Life Score}

The aggregated data for the 2527 patients in the effectiveness analysis set $(n=3144)$ who had paired QOL scores before and after silodosin administration are shown in Table 4. The mean QOL scores before and after administration were $4.2 \pm 0.9$ and $3.0 \pm 1.3$, respectively, indicating a significant improvement $(P<0.0001)$. In the subgroups of patients who had received an $\alpha_{1}$ blocker (tamsulosin or naftopidil) prior to silodosin treatment, similar significant improvements in the mean QOL were seen after switching $(P<0.0001)$.

\subsubsection{Improvement in Overactive Bladder Symptom Score}

Between 1117 and 2474 patients in the effectiveness analysis set $(n=3144)$ had data for each OABSS symptom before and after the administration of silodosin and a value for those parameters that was not 0 (Table 4). The mean total OABSS was $5.7 \pm 2.9$ at baseline and $4.5 \pm 2.7$ after silodosin, indicating a significant improvement $(P<0.0001)$. All four symptoms of the OABSS showed a significant improvement from baseline with silodosin $(P<0.0001)$. Significant improvements from baseline in total OABSS and all OABSS subscale scores were also seen in patients who had switched from tamsulosin or naftopidil $(P<0.0001)$.

\subsubsection{Improvement in Residual Urine Volume}

In the effectiveness analysis set, 1399 of the 3144 patients had residual urine volume data at baseline and after the administration of silodosin (Table 4). The mean residual urine volume at baseline was $55.0 \pm 68.6 \mathrm{~mL}$, decreasing to $37.8 \pm 49.7 \mathrm{~mL}$ during silodosin treatment $(P<0.0001)$. Similar reductions (improvements) in residual urine volume were seen in patients who had previously used tamsulosin or naftopidil before switching to silodosin $(P<0.0001)$.

\subsubsection{Improvement in the Total IPSS According to Patient Background Factors}

The aggregated data of the total IPSS before and after the administration of silodosin according to patient background factors are shown in Table 5. The mean total IPSS showed significant improvement during silodosin treatment in almost 


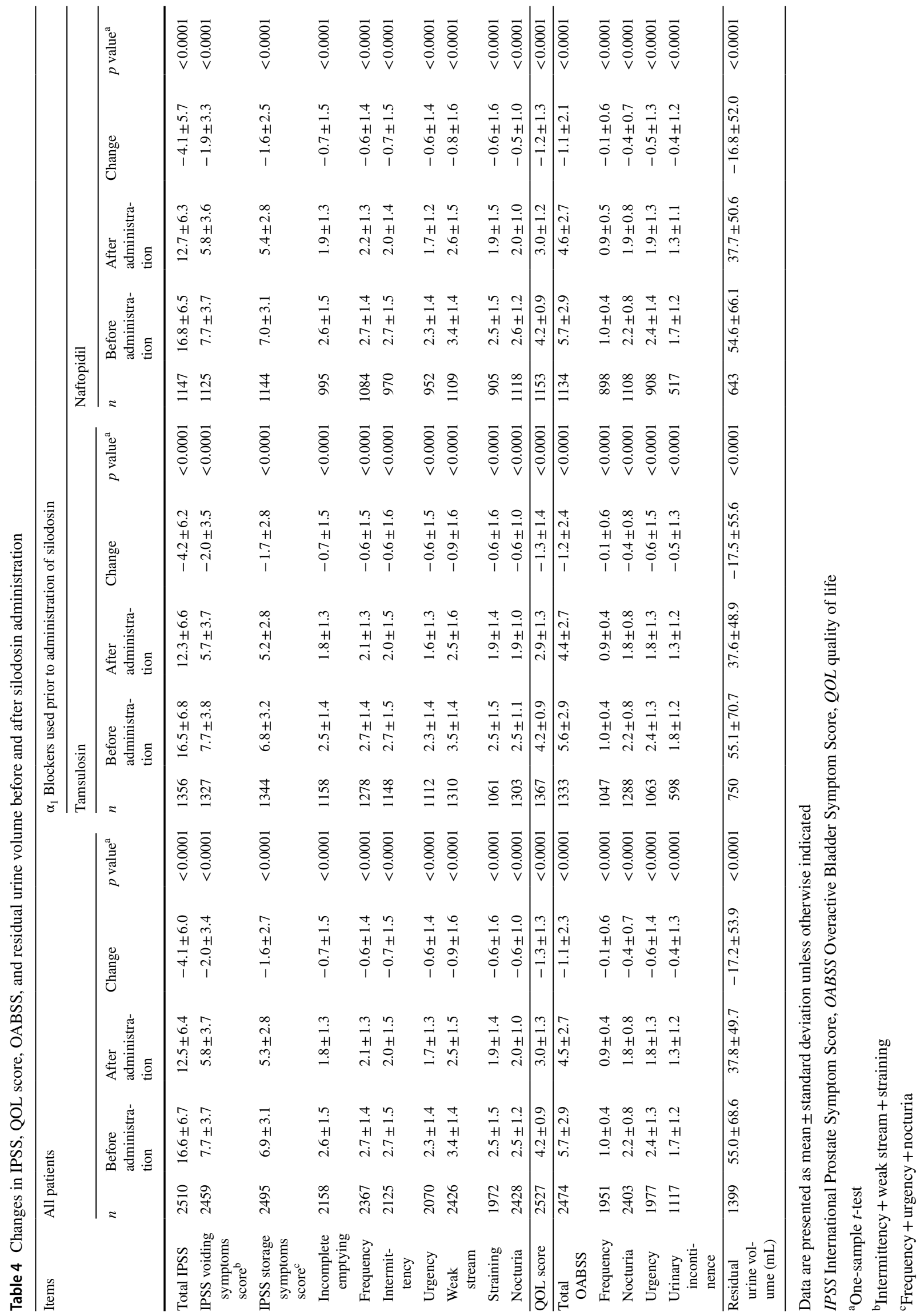


Table 5 Changes in total IPSS before and after silodosin administration in patient subgroups according to background factors

\begin{tabular}{|c|c|c|c|c|c|}
\hline Items and categories & $n$ & Before administration & After administration & Change & $p$ value $^{\mathrm{a}}$ \\
\hline All patients & 2510 & $16.6 \pm 6.7$ & $12.5 \pm 6.4$ & $-4.1 \pm 6.0$ & $<0.0001$ \\
\hline \multicolumn{6}{|l|}{ Age (years) } \\
\hline$<50$ & 8 & $13.3 \pm 6.0$ & $9.5 \pm 6.8$ & $-3.8 \pm 5.0$ & 0.0706 \\
\hline$\geq 50$ and $<60$ & 114 & $17.2 \pm 7.0$ & $12.8 \pm 6.7$ & $-4.4 \pm 5.1$ & $<0.0001$ \\
\hline$\geq 60$ and $<70$ & 652 & $16.6 \pm 6.7$ & $12.4 \pm 6.4$ & $-4.2 \pm 5.9$ & $<0.0001$ \\
\hline$\geq 70$ and $<80$ & 1153 & $16.5 \pm 6.6$ & $12.2 \pm 6.2$ & $-4.3 \pm 6.1$ & $<0.0001$ \\
\hline$\geq 80$ & 583 & $16.9 \pm 6.8$ & $13.2 \pm 6.9$ & $-3.7 \pm 5.9$ & $<0.0001$ \\
\hline Non-elderly patients: $<65$ & 350 & $16.6 \pm 6.8$ & $12.2 \pm 6.5$ & $-4.3 \pm 5.7$ & $<0.0001$ \\
\hline Elderly patients: $\geq 65$ & 2160 & $16.7 \pm 6.7$ & $12.5 \pm 6.4$ & $-4.1 \pm 6.0$ & $<0.0001$ \\
\hline \multicolumn{6}{|l|}{ BMI $\left(\mathrm{kg} / \mathrm{m}^{2}\right)$} \\
\hline$<18.5$ & 61 & $15.4 \pm 6.5$ & $12.3 \pm 6.2$ & $-3.1 \pm 5.0$ & $<0.0001$ \\
\hline$\geq 18.5$ and $<25.0$ & 1035 & $16.6 \pm 6.7$ & $12.5 \pm 6.6$ & $-4.1 \pm 6.0$ & $<0.0001$ \\
\hline$\geq 25.0$ and $<30.0$ & 425 & $16.7 \pm 7.0$ & $12.6 \pm 6.6$ & $-4.1 \pm 6.4$ & $<0.0001$ \\
\hline$\geq 30.0$ & 37 & $15.8 \pm 7.6$ & $11.5 \pm 7.2$ & $-4.2 \pm 7.4$ & 0.0013 \\
\hline Unknown/unlisted & 952 & $16.8 \pm 6.6$ & $12.5 \pm 6.2$ & $-4.3 \pm 5.8$ & $<0.0001$ \\
\hline \multicolumn{6}{|l|}{ Prostate volume (mL) } \\
\hline$<40$ & 1376 & $16.3 \pm 6.6$ & $12.4 \pm 6.3$ & $-3.9 \pm 6.0$ & $<0.0001$ \\
\hline$\geq 40$ & 837 & $17.1 \pm 6.8$ & $12.7 \pm 6.3$ & $-4.4 \pm 6.2$ & $<0.0001$ \\
\hline Unknown/unlisted & 297 & $16.8 \pm 7.0$ & $12.3 \pm 7.2$ & $-4.6 \pm 5.2$ & $<0.0001$ \\
\hline \multicolumn{6}{|l|}{$\mathrm{BPH}$ duration } \\
\hline$<3$ months & 0 & - & - & - & - \\
\hline$\geq 3$ months and $<1$ year & 447 & $16.2 \pm 6.5$ & $12.1 \pm 6.5$ & $-4.1 \pm 5.9$ & $<0.0001$ \\
\hline$\geq 1$ year and $<3$ years & 711 & $16.5 \pm 6.8$ & $11.9 \pm 6.2$ & $-4.6 \pm 6.0$ & $<0.0001$ \\
\hline$\geq 3$ years & 1257 & $16.7 \pm 6.7$ & $12.9 \pm 6.4$ & $-3.8 \pm 5.9$ & $<0.0001$ \\
\hline Unknown/unlisted & 95 & $18.9 \pm 7.1$ & $13.6 \pm 7.8$ & $-5.3 \pm 6.3$ & $<0.0001$ \\
\hline \multicolumn{6}{|l|}{ PSA (ng/mL) } \\
\hline$\leq 4.0$ & 1720 & $16.6 \pm 6.6$ & $12.5 \pm 6.3$ & $-4.1 \pm 5.9$ & $<0.0001$ \\
\hline$>4.0$ and $\leq 10.0$ & 495 & $16.4 \pm 6.5$ & $12.0 \pm 6.2$ & $-4.4 \pm 6.2$ & $<0.0001$ \\
\hline$>10.0$ & 70 & $17.2 \pm 7.9$ & $14.0 \pm 7.8$ & $-3.2 \pm 6.7$ & 0.0001 \\
\hline Unknown/unlisted & 225 & $17.3 \pm 7.2$ & $12.8 \pm 7.5$ & $-4.5 \pm 5.8$ & $<0.0001$ \\
\hline \multicolumn{6}{|c|}{$\alpha_{1}$ Blockers used prior to silodosin } \\
\hline Tamsulosin & 1356 & $16.5 \pm 6.8$ & $12.3 \pm 6.6$ & $-4.2 \pm 6.2$ & $<0.0001$ \\
\hline Naftopidil & 1147 & $16.8 \pm 6.5$ & $12.7 \pm 6.3$ & $-4.1 \pm 5.7$ & $<0.0001$ \\
\hline Other & 12 & $17.3 \pm 6.3$ & $13.3 \pm 5.5$ & $-3.9 \pm 3.9$ & 0.0051 \\
\hline \multicolumn{6}{|c|}{ Concomitant drugs for LUTS other than $\alpha_{1}$ blockers } \\
\hline Anticholinergics & 226 & $16.8 \pm 6.5$ & $13.6 \pm 6.6$ & $-3.2 \pm 5.1$ & $<0.0001$ \\
\hline $5 \alpha$-Reductase inhibitor & 244 & $15.3 \pm 6.5$ & $12.4 \pm 6.4$ & $-2.9 \pm 5.9$ & $<0.0001$ \\
\hline PDE5 inhibitor & 50 & $20.3 \pm 6.8$ & $15.2 \pm 5.0$ & $-5.1 \pm 5.8$ & $<0.0001$ \\
\hline$\beta_{3}$ Agonist & 171 & $17.1 \pm 6.8$ & $13.3 \pm 6.2$ & $-3.8 \pm 5.9$ & $<0.0001$ \\
\hline \multicolumn{6}{|l|}{ Total IPSS } \\
\hline Mild: 0-7 & 207 & $5.7 \pm 1.4$ & $6.7 \pm 4.3$ & $1.0 \pm 4.4$ & 0.0014 \\
\hline Moderate: 8-19 & 1465 & $13.8 \pm 3.3$ & $10.8 \pm 4.9$ & $-3.0 \pm 4.8$ & $<0.0001$ \\
\hline Severe: $20-35$ & 838 & $24.3 \pm 3.6$ & $16.9 \pm 6.7$ & $-7.4 \pm 6.5$ & $<0.0001$ \\
\hline \multicolumn{6}{|l|}{ QOL score } \\
\hline Mild: 0-1 & 0 & - & - & - & - \\
\hline Moderate: $2-4$ & 1647 & $14.6 \pm 5.9$ & $11.4 \pm 5.9$ & $-3.2 \pm 5.6$ & $<0.0001$ \\
\hline Severe: $5-6$ & 863 & $20.4 \pm 6.6$ & $14.5 \pm 7.0$ & $-5.9 \pm 6.3$ & $<0.0001$ \\
\hline \multicolumn{6}{|l|}{ Total OABSS } \\
\hline Mild: $\leq 5$ & 1312 & $14.0 \pm 5.8$ & $10.9 \pm 5.7$ & $-3.1 \pm 5.4$ & $<0.0001$ \\
\hline Moderate: 6-11 & 1079 & $19.2 \pm 6.3$ & $13.9 \pm 6.4$ & $-5.2 \pm 6.1$ & $<0.0001$ \\
\hline Severe: $\geq 12$ & 96 & $24.4 \pm 6.3$ & $17.8 \pm 9.0$ & $-6.6 \pm 8.8$ & $<0.0001$ \\
\hline
\end{tabular}


Table 5 (continued)

\begin{tabular}{lrllll}
\hline Items and categories & $n$ & Before administration & After administration & Change & $p$ value $^{\mathrm{a}}$ \\
\hline Residual urine volume $(\mathrm{mL})$ & & & & & \\
$<50$ & 1059 & $16.0 \pm 6.4$ & $12.2 \pm 6.3$ & $-3.7 \pm 5.9$ & $<0.0001$ \\
$\geq 50$ and $<100$ & 387 & $17.6 \pm 7.0$ & $12.6 \pm 6.6$ & $-5.0 \pm 6.8$ & $<0.0001$ \\
$\geq 100$ & 256 & $17.4 \pm 6.9$ & $12.8 \pm 6.3$ & $-4.6 \pm 6.2$ & $<0.0001$ \\
Unknown/unlisted & 808 & $16.8 \pm 6.8$ & $12.7 \pm 6.6$ & $-4.1 \pm 5.5$ & $<0.0001$ \\
\hline
\end{tabular}

Data are presented as mean \pm standard deviation unless otherwise indicated

$B M I$ body mass index, $B P H$ benign prostatic hyperplasia, IPSS International Prostate Symptom Score, LUTS lower urinary tract symptoms, OABSS Overactive Bladder Symptom Score, PDE phosphodiesterase, $P S A$ prostate-specific antigen, $Q O L$ quality of life

${ }^{\mathrm{a} O n e ~ s a m p l e ~ t-t e s t ~}$

all subgroups containing at least 50 patients $(P<0.001)$; the only exception was the group with mild symptoms (total IPSS $\leq 7)$ at baseline.

\section{Discussion}

The clinical guidelines for BPH in Japan recommend $\alpha_{1}$ blockers as a first-line drug therapy for BPH [3]. Although silodosin has been compared with tamsulosin or naftopidil in crossover or parallel studies [5-7], evidence concerning the effect of switching between $\alpha_{1}$ blockers is currently limited. We expected that this survey would demonstrate the specific patient characteristics suitable for switching to silodosin from other $\alpha_{1}$ blockers and the effect of the switching.

As for the safety profile, the incidence of ADRs with silodosin was $8.1 \%$ in this post-marketing surveillance, indicating a slightly lower rate compared with the aggregated results $(11.3 \%)$ [8] in the previous post-marketing surveys $[1,2]$. ADRs that occurred at an incidence of $\geq 0.5 \%$ included ejaculation disorder, retrograde ejaculation, diarrhoea, dizziness, and nasal congestion. No new safety signals were identified, as all of the common ADRs in our analysis were consistent with those in the previous surveys.

Consistent with the previous post-marketing surveys, the incidence of ADRs was significantly higher in non-elderly patients $(17.1 \%)$ than in elderly patients $(6.6 \%)$. A total of 271 patients developed ADRs, and the most common ADRs were ejaculation disorder $(n=52)$ and retrograde ejaculation $(n=47)$, events that are likely to be more frequent in non-elderly patients as shown in previous post-marketing data. The age-related incidence of ADRs based on 10-year increments was also investigated, and the result indicated a lower incidence of ADRs with more advancing age, as has been shown previously.

Since silodosin was launched, a number of new therapeutic agents for overactive bladder $(\mathrm{OAB})$ and $\mathrm{BPH}$ have become available, including anticholinergics, $5 \alpha$-reductase inhibitor, PDE5 inhibitor, and $\beta_{3}$ agonist. The incidence of ADRs in patients taking these agents concomitantly with silodosin $(7.6 \%)$ was not significantly different from that in silodosin recipients not taking concomitant BPH therapy (8.3\%). The incidence of ADRs according to the type of concomitant drugs was as follows: anticholinergics $7.7 \%$; $5 \alpha$-reductase inhibitor 6.9\%; PDE5 inhibitor $10.1 \%$; and $\beta_{3}$ agonist $5.6 \%$. Although the incidence of silodosin ADRs was slightly higher with concomitant use of a PDE5 inhibitor than with other drug classes, the number of patients receiving this combination was small $(n=69)$. Therefore, more data from a larger cohort of patients are needed.

As for the effectiveness profile, the mean change in total IPSS after switching to silodosin was $-4.1 \pm 6.0$, indicating a significant improvement in symptoms. Furthermore, significant improvements were observed in all effectiveness outcome measures, including the seven symptoms of the IPSS, the IPSS voiding symptom score, the IPSS storage symptom score, the QOL score, the total OABSS, the four symptoms of the OABSS, and residual urine volume. Similar improvements were observed in patients who had previously received tamsulosin or naftopidil before switching to silodosin, indicating no difference depending on the types of prior therapeutic drugs. The results suggested that switching to silodosin, which has high $\alpha_{1 \mathrm{~A}}$-receptor selectivity, could be a treatment option for patients with a low level of satisfaction with the treatment.

With the exception of patients who had mild symptoms (total IPSS $\leq 7$ ) at baseline, significant improvements in total IPSS were seen after switching in all subgroups containing at least 50 patients. Thus, switching to silodosin improved the total IPSS in most patients, regardless of background factors. Patients whose symptoms were of mild severity in the total IPSS during their initial $\alpha_{1}$ blocker treatment did not show further improvement after switching to silodosin. Therefore, total IPSS at the time of switching can be a predictor of the effect of the switching.

This study was conducted to investigate the effectiveness and safety of silodosin in patients with BPH who had not achieved satisfactory symptom control with other $\alpha_{1}$ blockers in routine clinical practice. The results confirmed the safety of silodosin, consistent with previous post-marketing 
surveys such as the drug use results survey, and showed there was no increase in the incidence of ADRs with the use of any type of concomitant drug for LUTS treatment. Moreover, significant improvements in effectiveness were observed in all outcome measures, including the IPSS, the QOL score and OABSS

Acknowledgements This article is an English translation of the article originally reported in the Japanese Journal of Urological Surgery (Takahashi H, Kubono S, Taneyama T, Kuramoto K, Mizutani H, Tanaka $\mathrm{N}$ and Yoshida M. Post-marketing surveillance of Urief ${ }^{\circledR} 2 \mathrm{mg}$ and $4 \mathrm{mg}$ tablets [silodosin]: SPLASH study-Surveillance in benign prostatic hyperplasia $[\mathrm{BPH}]$ patients with poor response to existing alpha-1 blockers-First report. 2017;30:327-340), not including the descriptions of (1) the effects of silodosin on the daily living of patients with $\mathrm{BPH},(2)$ patients' satisfaction with silodosin treatment assessed by the face scale questionnaire, (3) patients' evaluation for BPH medications that are favorable for continuous use, and (4) the correlation between the changes in the seven symptoms of the IPSS before and after silodosin administration and those in QOL scores. The authors would like to express their sincere gratitude to all physicians who cooperated with the specified drug use results survey of silodosin tablets and provided valuable data. We would also like to thank Ms. Catherine Rees and Go Kuratomi, Ph.D., of inScience Communications, Springer Healthcare, who edited and styled the manuscript for submission. This medical writing assistance was funded by Kissei Pharmaceutical Co., Ltd.

\section{Compliance with Ethical Standards}

Funding The survey was conducted through funding from Kissei Pharmaceutical Co., Ltd. and Daiichi Sankyo Co., Ltd.

Conflict of interest Hiroshi Takahashi, Shinichi Kubono, Takehiko Taneyama, and Kiyotoshi Kuramoto are employees of and hold stock options in Kissei Pharmaceutical Co., Ltd. Hideki Mizutani is an employee of and holds stock options in Daiichi Sankyo Co., Ltd. Noriko Tanaka is an employee of Daiichi Sankyo Co., Ltd. Masaki Yoshida has received grants from Astellas Pharma Inc.; consulting fees from Kyorin Pharmaceutical Co. Ltd. and Kissei Pharmaceutical Co. Ltd.; support for travel to meetings for the study, manuscript preparation and other purposes from Astellas Pharma Inc., Kyorin Pharmaceutical Co. Ltd., Kissei Pharmaceutical Co. Ltd. and Hisamitsu Pharmaceutical Co. Inc.; fees for participation in review activities from Astellas Pharma Inc., Kyorin Pharmaceutical Co. Ltd., Kissei Pharmaceutical Co. Ltd. and Hisamitsu Pharmaceutical Co. Inc.; payment for writing or reviewing the manuscript from Astellas Pharma Inc., Kyorin Pharmaceutical Co. Ltd., Kissei Pharmaceutical Co. Ltd. and Hisamitsu Pharmaceutical Co. Inc.; and payment for lectures, including service on speakers bureaus, from Astellas Pharma Inc., Kyorin Pharmaceutical Co. Ltd., Kissei Pharmaceutical Co. Ltd. and Hisamitsu Pharmaceutical Co. Inc.

Data availability The datasets generated and analysed during the current study are not publicly available because our dataset includes identified individual participants data
Research involving human participants This survey was conducted in accordance with good post-marketing study practice in Japan.

Informed consent According to good post-marketing study practice in Japan, informed consent was not required for this post-marketing study. As such, informed consent was not obtained from individual participants included in the study.

Open Access This article is distributed under the terms of the Creative Commons Attribution-NonCommercial 4.0 International License (http://creativecommons.org/licenses/by-nc/4.0/), which permits any noncommercial use, distribution, and reproduction in any medium, provided you give appropriate credit to the original author(s) and the source, provide a link to the Creative Commons license, and indicate if changes were made.

\section{References}

1. Iwasa Y, Mizutani H, Endo H, Kuroyanagi K, Shiozawa T, Nakano Y. Urief capsules (generic name: silodosin) post marketing surveillance-drug use-results survey (article in Japanese). Med Cons New Remed. 2010;47:304-17.

2. Iwasa Y, Mizutani H, Endo H, Kuroyanagi K, Taneyama T. Urief (generic name: silodosin) post marketing surveillance-specified drug use-results survey on long-term use (article in Japanese). Med Cons New Remed. 2013;50:763-75.

3. Association The Japanese Urological. Clinical guideline for benign prostatic hyperplasia. Tokyo: RichHill Medical Inc.; 2011.

4. Iwasa Y, Taneyama T, Takahashi H, et al. Searching for background factors influencing the improvement effect of silodosin on lower urinary tract symptoms associated with benign prostatic hyperplasia-analysis of two post-marketing surveillance studies (article in Japanese). Jpn J Urol Surg. 2014;27:1663-70.

5. Kawabe K, Yoshida M, Homma Y, Silodosin Clinical Study Group. Silodosin, a new alpha1A-adrenoceptor-selective antagonist for treating benign prostatic hyperplasia: results of a phase III randomized, placebo-controlled, double-blind study in Japanese men. BJU Int. 2006;98:1019-24.

6. Miyakita H, Yokoyama E, Onodera Y, et al. Short-term effects of crossover treatment with silodosin and tamsulosin hydrochloride for lower urinary tract symptoms associated with benign prostatic hyperplasia. Int J Urol. 2010;17:869-75.

7. Shirakawa T, Haraguchi T, Shigemura K, et al. Silodosin versus naftopidil in Japanese patients with lower urinary tract symptoms associated with benign prostatic hyperplasia: a randomized multicenter study. Int J Urol. 2013;20:903-10.

8. Kissei Pharmaceutical Co., Ltd., Daiichi Sankyo Co., Ltd. Package insert of URIEF $^{\circledR}$ tab. $2 \mathrm{mg} / 4 \mathrm{mg}$ and URIEF ${ }^{\circledR}$ OD tab. $2 \mathrm{mg} / 4 \mathrm{mg}$, Seventh Edition [revised Aug 2016] (in Japanese); 2016 . 